\title{
Transport Model Studies of the Baryon-Rich Quark-Gluon Plasma formed in Heavy Ion Collisions
}

\section{Lie-Wen Chen}

Department of Physics, Shanghai Jiao Tong University, Shanghai 200240, China

E-mail: lwchenesjtu.edu.cn

\section{Che Ming Ko*}

Cyclotron Institute and Department of Physics and Astronomy, Texas A\&M University, College

Station, Texas 77843-3366

E-mail: ko@comp.tamu.edu

Wei Liu ${ }^{\dagger}$

Cyclotron Institute, Texas A\&M University, College Station, Texas 77843-3366

E-mail: weiliu@comp.tamu.edu

\section{Ben-Wei Zhang}

Institute of Particle Physics, Huazhong Normal University, Wuhan 430079, China

E-mail: bwzhang@iopp.ccnu.edu.cn

Heavy ion collisions in low energy runs at Relativistic Heavy Ion Collider (RHIC) and at future Facility for Antiproton and Ion Research (FAIR) in Germany are expected to produce a quarkgluon plasma that has a finite baryon chemical potential, allowing thus the possibility to study the location of the critical endpoint in the QCD phase diagram. In this talk, using a multiphase transport model, that includes interactions in both initial partonic and final hadronic matters and the transition between these two phases of matter, we discuss the effects of partonic interactions on observables such as the elliptic flow that have played essential roles in studying the properties of the net baryon free quark-gluon plasma produced in heavy ion collisions at higher energies at RHIC. Also, we study the effect of density fluctuations due to a first-order transition between the quark-gluon plasma and hadronic matter on fluctuations of hadron mean transverse momentum and produced deuteron number as well as on two-pion correlations. We further discuss the possibility of studying the mechanism of charm energy loss in the baryon-rich quark-gluon plasma and the properties of phi mesons in hot-dense matter produced in these collisions.

5th International Workshop on Critical Point and Onset of Deconfinement - CPOD 2009,

June 08 - 122009

Brookhaven National Laboratory, Long Island, New York, USA

\footnotetext{
${ }^{*}$ Speaker.

${ }^{\dagger}$ Present address: Department of Finance, Texas A\&M University, College Station, Texas 77843-4218
} 


\section{Introduction}

Many observables have been proposed as possible signatures for the deconfined plasma of quarks and gluons that is produced during initial stage of ultra-relativistic heavy ion collisions. These include enhanced production of dileptons of intermediate invariant masses [1] and baryons made of multi-strange quarks [2], increased emission duration [3, 4], suppressed production of charmonia [5], large anisotropic flows of hadrons [6], quenching of minijets with large transverse momenta [7], and scaling of hadron elliptic flows according to their constituent quark content [8]. Most of these observables have been studied during past many years in experiments at RHIC involving $\mathrm{Au}+\mathrm{Au}$ collisions at center-of-mass energies $\sqrt{s_{N N}}=62,130$, and $200 \mathrm{GeV}$. Studying these signatures using various theoretical models, such as the statistical model $[9,10]$, the hydrodynamic model [11, 12, 13], the transport model [14, 15, 16, 17, 18, 19, 20, 21, 22, 23], the quark coalescence model [24, 25, 26, 27], and the perturbative QCD approach [28, 29], has provided convincing evidence that the quark-gluon plasma QGP has indeed been produced in these collisions. Moreover, these studies have indicated that the quark-gluon plasma produced at RHIC is strongly interacting with transport coefficients very different from those given by the perturbative QCD [30].

Heavy ion collisions at energies much higher than that at RHIC will soon be available at the Large Hadron Collider (LHC), and it is expected that the produced quark-gluon plasma will have even higher temperature and smaller baryon chemical potential than that produced in heavy ion collisions at RHIC. On the other hand, a quark-gluon plasma with finite baryon chemical potential is expected to be produced from heavy ion collisions in low energy runs at RHIC and at future FAIR. Although lattice QCD calculations have not been able to address the properties of quarkgluon plasma at finite baryon chemical potential, theoretical models such as the PNJL model have shown that the smooth crossover from the quark-gluon plasma to the hadronic matter transition predicted by lattice gauge calculations for zero baryon chemical potential would change to a firstorder phase transition when the baryon chemical potential becomes sufficient large [31]. Heavy ion collisions in low energy runs at RHIC and at FAIR thus allow the possibility to study the location of the critical endpoint in the QCD phase diagram at which the first-order phase transition changes to a smooth crossover. It is therefore of great interest to make predictions for above mentioned observables in these collisions based on what we have learnt from heavy ion collisions at high energy runs at RHIC. Using a multiphase transport (AMPT) model, which has been quite useful in understanding experimental results at RHIC, we have carried out such a study. In particular, we have made predictions on the rapidity distributions of various hadrons, their elliptic flows, twopion correlation functions, and the effects of a first-order quark-gluon plasma to hadronic matter phase transition in $\mathrm{Au}+\mathrm{Au}$ collisions at center-of-mass energy of $\sqrt{s_{N N}}=7 \mathrm{GeV}$ (corresponding to a beam energy of about $E_{\text {beam }} \approx 25 \mathrm{GeV} /$ nucleon in the laboratory system with fixed target), that is available in the low-energy runs at RHIC and at FAIR.

\section{A multiphase transport model}

Before presenting predicted results, we review briefly the AMPT model $[14,15,16,32,33$, $34,35]$. It is a hybrid model that uses minijet partons from hard processes and strings from soft 
processes in the heavy ion jet interaction generator (HIJING) model [36] as initial conditions for modeling heavy-ion collisions at ultra-relativistic energies. Time evolution of resulting minijet partons, which are largely gluons, is described by Zhang's parton cascade (ZPC) model [37]. At present, this model includes only parton-parton elastic scatterings with an in-medium cross section given by the perturbative QCD, i.e.,

$$
\frac{d \sigma_{p}}{d t} \simeq \frac{9 \pi \alpha_{s}^{2}}{2\left(t-\mu^{2}\right)^{2}}, \quad \sigma_{p} \simeq \frac{9 \pi \alpha_{s}^{2}}{2 \mu^{2}} \frac{1}{1+\mu^{2} / s},
$$

where $\alpha_{s}$ is the strong coupling constant and is taken to have a value of 0.47 , and $s$ and $t$ are the usual Mandelstam variables for squared center-of-mass energy and four momentum transfer, respectively. The effective screening mass $\mu$ depends on the temperature and density of the partonic matter but is taken as a parameter in ZPC for fixing the magnitude and angular distribution of the parton scattering cross section. After minijet partons stop interacting, they are combined with their parent strings, as in the HIJING model with jet quenching, to fragment into hadrons using the Lund string fragmentation model as implemented in the PYTHIA program [38]. The final-state hadronic scatterings are further modeled by a relativistic transport (ART) model $[39,40]$. This default AMPT model has been quite successful in describing measured rapidity distributions of charged particles, particle to antiparticle ratios, and spectra of low transverse momentum pions and kaons in heavy ion collisions at the Super Proton Synchrotron (SPS) and RHIC [15]. It has also been useful in understanding the production of $J / \psi[32]$ and multistrange baryons [35] in these collisions.

Since the initial energy density in ultra-relativistic heavy ion collisions is expected to be much larger than the critical energy density at which the hadronic matter to quark-gluon plasma transition would occur [16, 32, 41], the AMPT model has been extended to allow the initially excited strings to melt into partons [42]. In this version, hadrons that would have been produced from the HIJING model are converted to their valence quarks and/or antiquarks. Interactions among these partons are again described by the ZPC parton cascade model. Because inelastic scatterings are not included in the current version of the ZPC model, only quarks and antiquarks from melted strings are present in the partonic matter. The species independence of the cross section used in the ZPC model compensates, however, for the absence of gluons in the early stage.

The transition from the partonic matter to the hadronic matter in the AMPT with string melting is achieved using a coordinate-space quark coalescence model, i.e., two nearest quark and antiquark are combined into mesons and three nearest quarks or antiquarks are combined into baryons or antibaryons that are closest to the invariant masses of these parton combinations. This coalescence model is somewhat different from the ones that are based on the overlap of hadron quark wave functions with the quark distribution functions in the partonic matter and used extensively for studying the production of hadrons with intermediate transverse momenta [24, 25, 26]. The finalstate scatterings of produced hadrons from quark coalescence are again described by the ART model.

Using parton scattering cross sections of $6-10 \mathrm{mb}$, the AMPT model with string melting is able to reproduce the centrality and transverse momentum (below $2 \mathrm{GeV} / c$ ) dependence of hadron elliptic flows [42] and higher-order anisotropic flows [43] as well as the pion interferometry [44] measured in $\mathrm{Au}+\mathrm{Au}$ collisions at $\sqrt{s_{N N}}=130 \mathrm{GeV}$ at RHIC $[45,46,47]$. It has also been used to study the kaon interferometry [48] in these collisions as well as many other observables at $\sqrt{s_{N N}}=$ 
$200 \mathrm{GeV}$, such as the pseudorapidity [49], system size [50, 51], and flavor [52, 53] dependence of anisotropic flows.

\section{Trajectories of central heavy ion collisions in the QCD phase diagram}
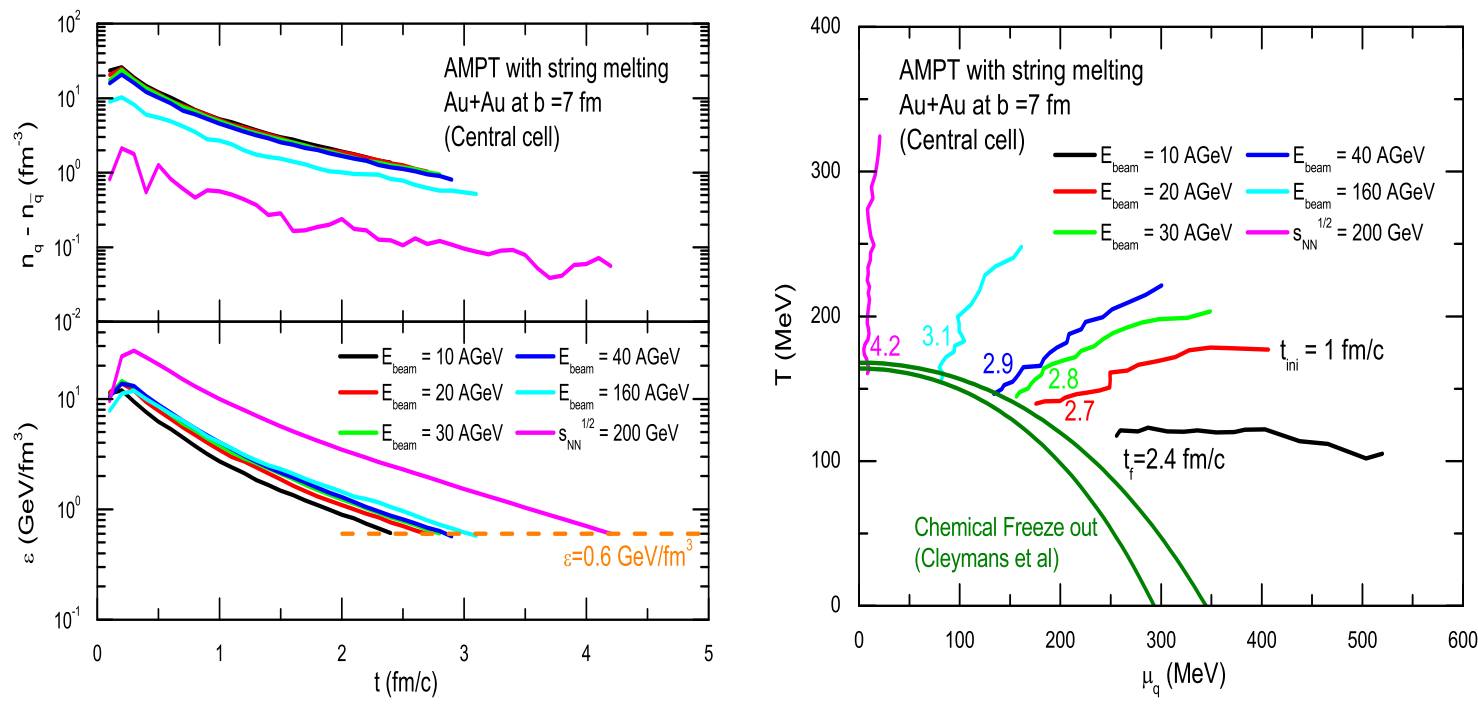

Figure 1: (Color online) Left window: Time evolution of net quark and energy densities. Right window: Trajectories of central heavy ion collisions in the QCD phase diagram of temperature and net quark chemical potential.

Using the AMPT model with string melting, we have studied the time evolution of net quark density and energy density of produced partonic matter in relativistic heavy ion collisions. This is shown in the left window of Fig. 1 for the central cell in the produced matter, which is taken to have a transverse radius of $1 \mathrm{fm}$ and a longitudinal dimension of $5 \%$ of the time after the two nuclei have fully overlapped in the longitudinal direction. If we assume that these partons are in thermal equilibrium, their temperature can then be determined. The time evolution of the temperature and baryon chemical potential of produced partonic matter is shown in the right window of Fig. 1. It is seen that the duration of the partonic stage decreases slightly with collision energy, about $4.2 \mathrm{fm} / \mathrm{c}$ in RHIC high energy runs and less than $3 \mathrm{fm} / c$ at lower energies and at FAIR. Although the baryon chemical potential (given by $3 \mu_{q}$ ) in RHIC high energy heavy ion collisions is small, it increases significantly as the collision energy decreases.

\section{Rapidity distributions}

The number of particles produced in a heavy ion collision provides valuable information on the energy density of the matter formed during the initial stage. Shown in Fig. 2 are the rapidity distributions of identified hadrons in central $(b=0 \mathrm{fm}) \mathrm{Au}+\mathrm{Au}$ collisions at $\sqrt{s_{N N}}=7 \mathrm{GeV}$. The total multiplicity density at midrapidity is about 450 , which is about a factor of three lower than that in high energy runs at RHIC. This is consistent with an initial energy density of the central 


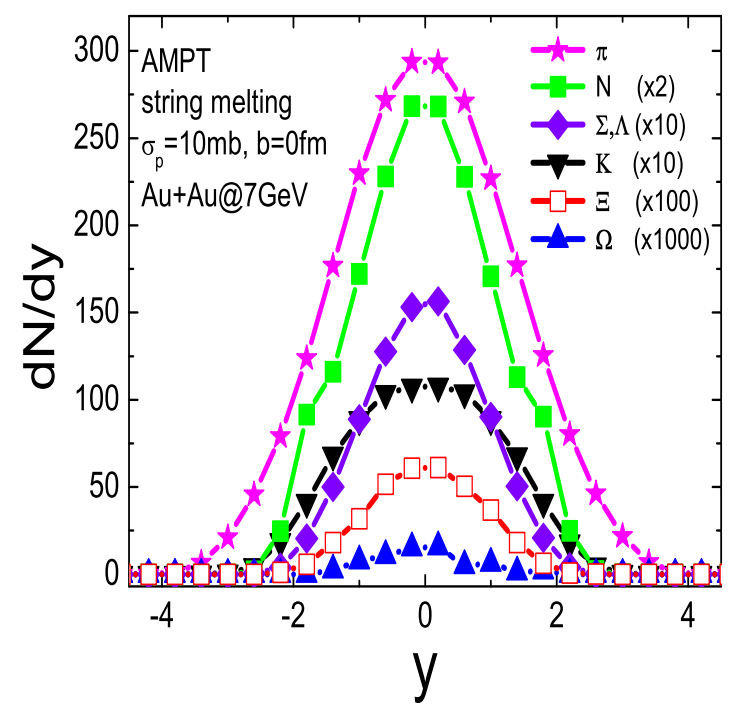

Figure 2: (Color online) Rapidity distributions of identified hadrons in central Au+Au collisions at $\sqrt{s_{N N}}=$ $7 \mathrm{GeV}$ from the AMPT model with string melting.

cell, which is about $10 \mathrm{GeV} / \mathrm{fm}^{3}$, that is also about a factor of three lower than that in high energy runs at RHIC. On the other hand, the net baryon rapidity density at midrapidity in central heavy ion collisions at this lower energy is about 135 and is almost half of that for pions, which is in contrast to heavy ion collisions in high energy runs at RHIC where the net baryon rapidity density at midrapidity is less than 10. For strange hadrons, more hyperons than kaons are produced in heavy ion collisions at low energies as a result of the large baryon chemical potential. This is again in sharp contrast to what was observed in high energy runs at RHIC, where the number of produced kaons is order of magnitude larger than that of hyperons. The large baryon chemical potential also leads to a yield of multistrange baryons in these collisions that is comparable to that in higher energy collisions at RHIC .

\section{Anisotroic flows}

In non-central heavy ion collisions, the spatial anisotropy of initially produced matter in the transverse plane is converted to the momentum anisotropy of produced particles. Results for $\mathrm{Au}+\mathrm{Au}$ collisions at $\sqrt{s_{N N}}=7 \mathrm{GeV}$ and impact parameter $b=7 \mathrm{fm}$ with a parton scattering cross section of $10 \mathrm{mb}$ are shown in Fig. 3. As shown in the left window, the quark elliptic flows at low transverse momentum follow the mass ordering with the charm quark elliptic flow much smaller than that of light and strange quarks. Their values become, however, similar at high transverse momentum and are comparable to those in high energy runs at RHIC. The elliptic flows of identified hadrons, such as the pion, kaon, nucleon, and various hyperons, are shown in the middle window of Fig. 3 using the scaled elliptic flow and transverse momentum, i.e, both are divided by the number of constituent quarks in a hadron. It is seen that the constituent quark number scaling expected from the naive coalescence model, in which only quarks with same momentum can coalescence into a hadron, is not exactly satisfied as the coalescence model used in the AMPT model for de- 

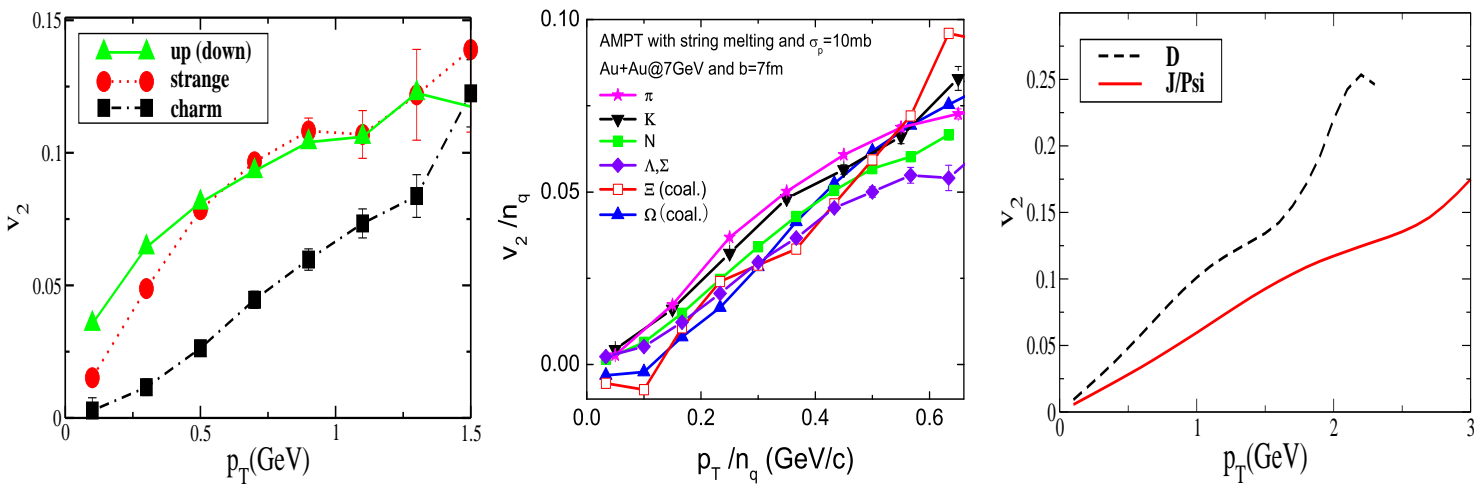

Figure 3: (Color online) Differential elliptic flows of quarks (left window), light (middle window) and heavy (right window) hadrons in central $\mathrm{Au}+\mathrm{Au}$ collisions at $\sqrt{s_{N N}}=7 \mathrm{GeV}$.

scribing the hadronization of the partonic matter is based on the coordinate-space consideration as described in Section 2. In the right window of Fig. 3, the elliptic flows of charmed meson and charmonium are shown. For charmed mesons, their elliptic flow $v_{2, D}\left(p_{T}\right)$ at transverse momentum $p_{T}$ is obtained using the quark coalescence or recombination model of Ref.[27], i.e.,

$$
v_{2, D}\left(p_{T}\right) \approx v_{2, c}\left(\left(m_{c} / m_{D}\right) p_{T}\right)+v_{2, q}\left(\left(m_{q} / m_{D}\right) p_{T}\right) .
$$

In the above, $v_{2, c}$ and $v_{2, q}$ are elliptic flows of charm and light quarks, respectively; while $m_{D}$, $m_{c}=1.5 \mathrm{GeV}$, and $m_{q}=300 \mathrm{MeV}$ are, respectively, the masses of charmed meson, charm quark, and light quark. Because of the much larger charm quark mass than those of light quarks, the elliptic flows of charmed mesons are close to that of heavy quarks. Eq.(5.1) can be generalized to heavy mesons with hidden charm, i.e., the charmonium $J / \psi$ that consists of a charm quark and its antiquark. Its elliptic flow at $p_{T}$ is then twice that of charm quark at $p_{T} / 2$ and is also shown in the right window of Fig. 3.

The elliptic flows of heavy mesons have already been studied in high energy runs at RHIC via measurement of their decay electrons $[54,55]$. The observed large value in Au+Au collisions at $\sqrt{s_{N N}}=200 \mathrm{GeV}$ is consistent with large elliptic flows of heavy quarks, particular that of charmed quarks as shown in Refs.[52, 56] based on the quark coalescence model. Without heavy quark elliptic flow, resulting heavy meson elliptic flow would be much smaller as shown in Ref.[57]. Studying heavy meson elliptic flow in low energy runs at RHIC and at FAIR is thus very useful for understanding the dynamics of heavy quarks in the partonic matter with finite baryon chemical potential.

As in heavy ion collisions from high energy runs at RHIC, the elliptic flows in heavy ion collisions from low-energy runs at RHIC and at FAIR would be significantly reduced if the partonic matter is not produced during the initial stage of the collisions.

\section{Effects of density fluctuation due to a first-order phase transition}

As mentioned previously, the baryon-rich partonic matter produced in heavy ion collisions from RHIC low-energy runs and at FAIR is expected to undergo a first order phase transition to 

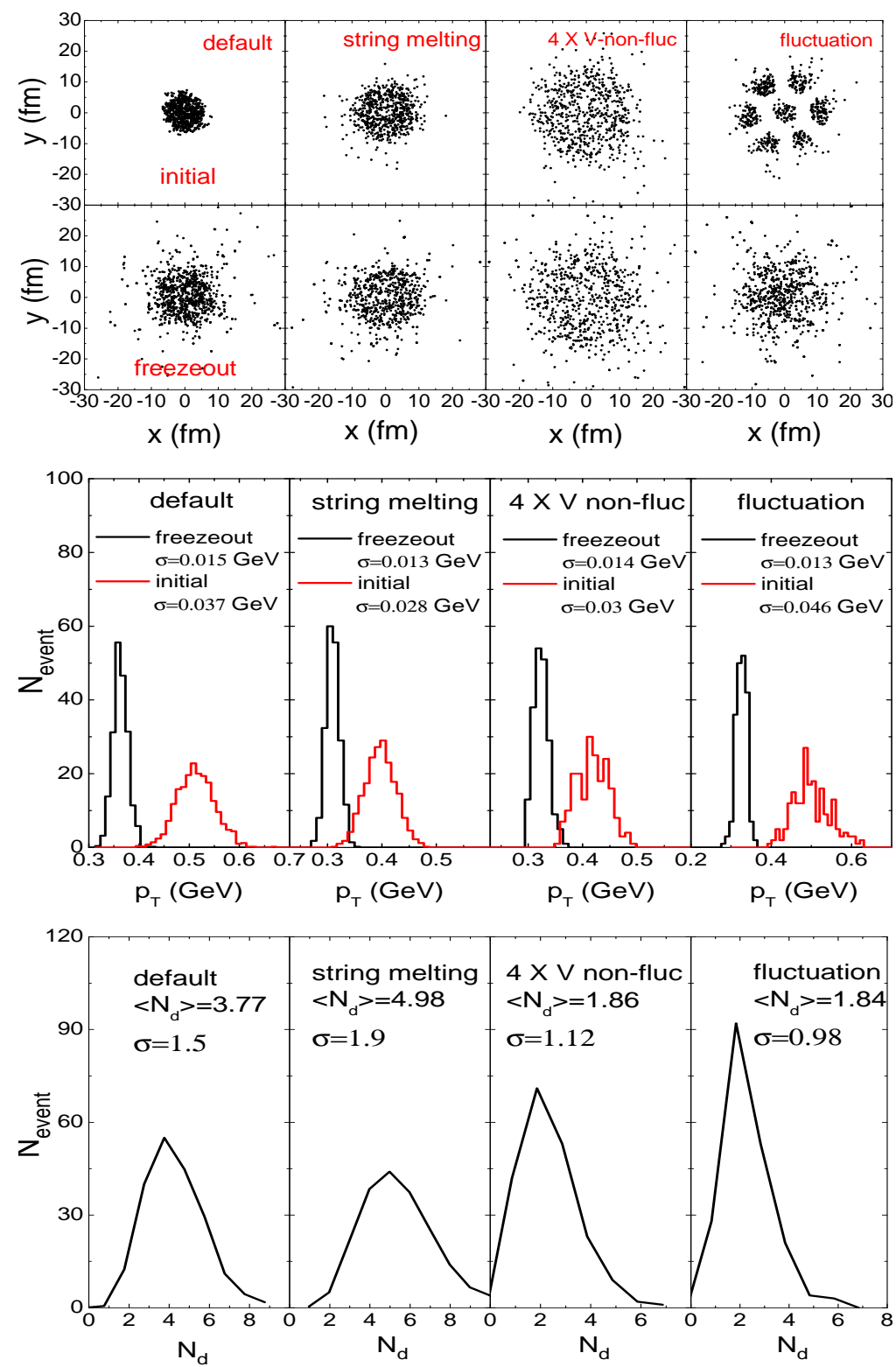

Figure 4: (Color online) Top window: Initial (upper panel) and final (lower panel) hadronic spatial distributions in different scenarios. Middle window: Fluctuation of hadron mean transverse momentum. Bottom window: Fluctuation of final deuteron number. All are for central $\mathrm{Au}+\mathrm{Au}$ collisions at $\sqrt{s_{N N}}=7 \mathrm{GeV}$.

the hadronic matter, instead of the smooth crossover transition of the quark-gluon plasma with low baryon chemical potential that is produced in RHIC high energy runs. To model the effect due to a first-order quark-gluon plasma to hadronic matter phase transition, which is at present absent in the AMPT model, a large density fluctuation is introduced in the hadronic matter formed immediately after hadronization. Specifically, we assume that the hadronic matter after a first-order phase transition consists of clusters of various sizes. This is achieved by redistributing hadrons produced in the AMPT with string melting in a volume that is four times larger but keeping their momenta unchanged. Furthermore, the average number of clusters is taken to be five and follows a Pois- 
son distribution as in the analysis of the nuclear matter gas-liquid phase transition in low-energy heavy ion collisions [58]. An example of the hadron spatial distribution is shown in the upper right panel of the top window in Fig. 4, and it is compared with those in the default AMPT, AMPT with string melting, and AMPT with string melting together with four times larger initial hadronic matter volume but without fluctuation, shown in other panels of the top window. The final hadronic spatial distributions in these four scenarios are shown in the lower panels of the top window and are seen to be different. As probes of these different scenarios, we have considered the fluctuation of final hadron mean transverse momentum (middle window) as well as that of final deuteron yield (bottom window), which is obtained by the coalescence model using the proton and neutron phase distributions at freeze out [59]. It is seen that the root mean square fluctuation of final hadron mean transverse momentum is smaller than that of initial hadron mean transverse momentum, and its value is similar in all scenarios. As a result, the mean transverse momentum fluctuation is not sensitive to the initial density fluctuation of produced hadrons. For the deuteron number fluctuation, increasing the initial volume of the hadronic matter reduces the final deuteron yield compared to those from the default AMPT and AMPT with string melting. The final deuteron number is, however, essentially independent of whether there is an initial fluctuation in the hadronic matter density. Although the initial density fluctuation is destroyed by final-state hadronic scatterings, it cannot exist without an increasing initial volume of hadronic matter as a result of the first-order phase transition.

\section{Two-pion correlations}

Particle interferometry based on the Hanbury-Brown Twiss (HBT) effect can provide information not only on the spatial extent of the emission source but also on its expansion velocity and emission duration $[3,4,60,61]$. In particular, the long emission time as a result of phase transition from the quark-gluon plasma to the hadronic matter in relativistic heavy ion collisions is expected to lead to an emission source which has a much larger radius in the direction of the total transverse momentum of detected two particles $\left(R_{\text {out }}\right)$ than that $\left(R_{\text {side }}\right)$ perpendicular to both this direction and the beam direction $\left(R_{\text {long }}\right)$. Although the extracted ratio $R_{\text {out }} / R_{\text {side }}$ from a Gaussian fit to the measured two-pion correlation function in $\mathrm{Au}+\mathrm{Au}$ collisions at $\sqrt{s_{N N}}=130 \mathrm{GeV}$ is close to one $[62,63,64]$, the source function extracted from the imaging method seems to show a longer tail in the out direction compared to other directions [65]. The small value of $R_{\text {out }} / R_{\text {side }}$ has been attributed to strong space-time and momentum correlations in the emission source [66]. Since the quark-gluon plasma produced in RHIC low-energy runs and at FAIR is expected to undergo a first-order phase transition, the emission source is expected to also have a large radius in the out direction.

Using the emission function obtained from the AMPT model with string melting and a parton scattering cross section of $10 \mathrm{mb}$ for central $(b=0 \mathrm{fm}) \mathrm{Au}+\mathrm{Au}$ collisions at $\sqrt{s_{N N}}=7 \mathrm{GeV}$, we have evaluated the correlation function $C_{2}(\mathbf{Q}, \mathbf{K})$ in the longitudinally comoving frame using the program Correlation After Burner [67] that takes into account final-state strong and Coulomb interactions between two charged pions. In Fig. 5, we show the calculated correlation function including final-state Coulomb interactions for midrapidity $(-0.5<y<0.5)$ charged pions with transverse momentum $125<p_{\mathrm{T}}<225 \mathrm{MeV} / c$ as a function of invariant momentum in the three 


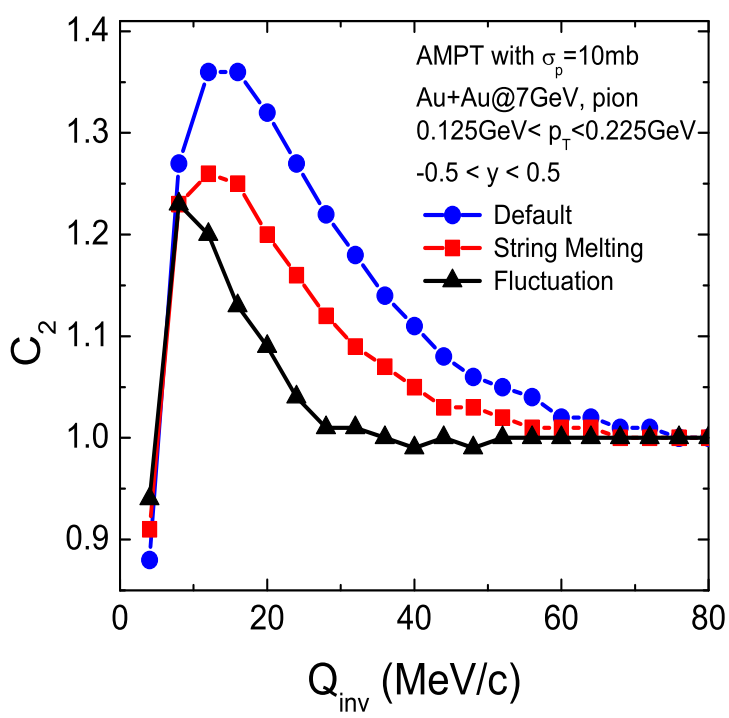

Figure 5: (Color online) Two-pion correlation functions in central Au+Au collisions at $\sqrt{s_{N N}}=7 \mathrm{GeV}$.

scenarios of default AMPT (filled circles), AMPT with string melting (filled squares), and AMPT with density fluctuation after hadronization (filled triangles). It is seen that the correlation function becomes narrower when a partonic matter is formed, and the width is further reduced if the initial volume of the hadronic matter after phase transition becomes larger when the phase transition is first-order.

\section{Charm suppression}

The partonic matter with a finite baryon chemical potential that is expected to be formed in RHIC low energy runs and at FAIR offers a possibility to test the idea recently introduced in Ref.[56] that the large charm quark elliptic flow observed in high energy runs at RHIC is a result of resonance scattering between charm quark and anti-light quarks in the produced partonic matter. Specifically, the scattering cross section between a charm quark and an light antiquark or a charm antiquark and a light quark is given by

$$
\sigma_{\bar{c} q \rightarrow \bar{c} q}\left(s^{1 / 2}\right)=\frac{1}{9} \frac{2 J+1}{4} \frac{\pi}{k^{2}} \frac{\Gamma_{D}^{2}}{\left(s^{1 / 2}-m_{D}\right)^{2}+\Gamma_{D}^{2} / 4}
$$

in terms of the charm meson mass $m_{D}$, width $\Gamma_{D}$ and spin $J$. Taking $m_{D}=2 \mathrm{GeV}, \Gamma_{D}=0.3-$ $0.5 \mathrm{GeV}$, charm quark mass $m_{c}=1.5 \mathrm{GeV}$, light quark mass $m_{q}=5-10 \mathrm{MeV}$ and including scalar, pseudoscalar, vector and axial vector charmed mesons gives a peak cross section of about $6 \mathrm{mb}$ at the charmed meson mass. The drag coefficient for charm quark or antiquark in a baryonfree quark-gluon plasma can then be calculated using the scattering amplitude $\mathscr{M}$ of the resonance scattering via

$$
\gamma(|\mathbf{p}|, T)=\sum_{i}\left(\left\langle\overline{|\mathscr{M}|^{2}}\right\rangle-\left\langle\overline{|\mathscr{M}|^{2}} \mathbf{p} \cdot \mathbf{p}^{\prime}\right\rangle /|\mathbf{p}|^{2}\right) .
$$

In the above, $\mathbf{p}$ and $\mathbf{p}^{\prime}$ are, respectively, the momenta of the heavy quark before and after a collision. Since the resonance scattering cross section is isotropic, it leads to a drag coefficient of 
$\gamma \sim 0.16 c / \mathrm{fm}$ which is about a factor of three larger than that given by the pQCD $t$-channel diagrams. Since there are about equal numbers of light quark and antiquarks in the quark-gluon plasma produced in high energy runs at RHIC, charm and anticharks thus lose same appreciable energy when traversing through the baryon-free quark-gluon plasma.

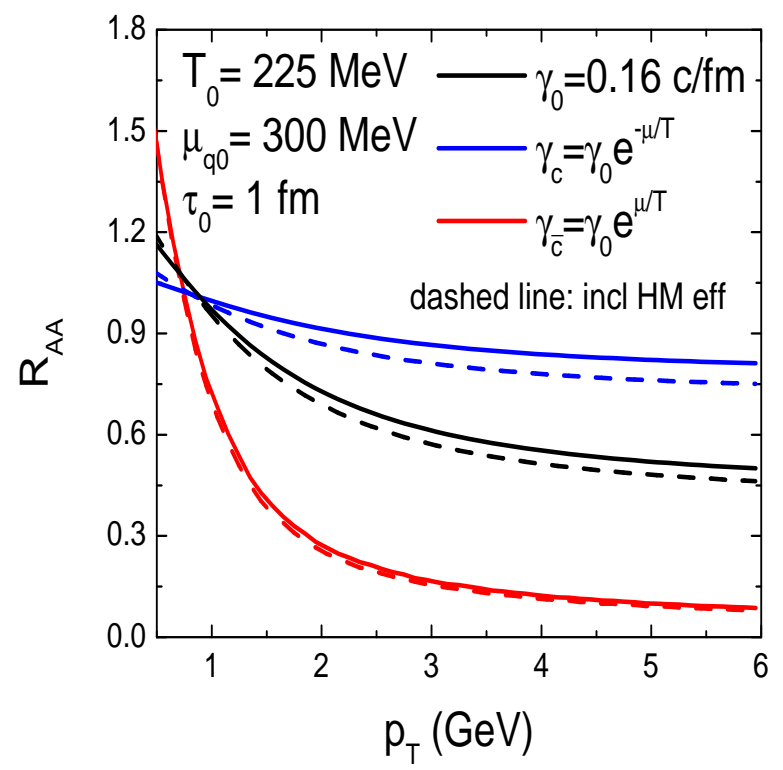

Figure 6: (Color online) Nuclear suppression factor for charmed meson and anticharmed mesons in baryonrich quark-gluon plasma.

For heavy ion collisions in RHIC low-energy runs and at FAIR, resonance scattering affects charm and anticharm quarks differently as there are more light quarks than light antiquarks in the baryon-rich quark-gluon plasma produced in these collisions, with the quark drag coefficients decreased by the factor $e^{-\mu / T}$ for charm quarks and increased by the factor $e^{\mu / T}$ for anti-charm quarks. For $\mathrm{Au}+\mathrm{Au}$ collisions at $\sqrt{s_{N N}}=7 \mathrm{GeV}$, the initial temperature and baryon chemical potential of produced quark-gluon plasma are $T_{0}=225 \mathrm{MeV}$ and $\mu_{0}=300 \mathrm{MeV}$ according to the AMPT model as shown in Fig.1. Taking the quark-gluon plasma to have a spherical shape with an initial radius $R_{0}=7 \mathrm{fm}$, the time evolution of the radius can then be determined from the time evolution of temperature and baryon chemical potential given in Fig. 1 if we assume that the entropy of the quark-gluon plasma is conserved during its expansion. For the distribution of initially produced charm and anti-charm quarks, their momentum spectra are generated by the PYTHIA program [68] and they are assumed to be produced uniformly inside the quark-gluon plasma. As charm and anti-charm quarks traverse through the expanding quark-gluon plasma, they loose their momentum according to $d p / d t \approx-\gamma p$. The resulting nuclear modification factors $R_{A A}$ for charm and anti-charm quarks, defined as the ratios of their final to initial momentum spectra are shown in Fig.6. It is seen that the nuclear modification factor for anti-charm quarks is much smaller than that for charm quarks. This result is not much affected by final-state hadronic reactions as also shown in the figure. We therefore expect that the momentum spectrum of charmed mesons will be less quenched than that of anti-charmed mesons from heavy ion collisions in RHIC low-energy runs and at FAIR, which thus provides a very interesting opportunity to study the dynamics of charm and anti-charm quarks in the net baryon-rich quark-gluon plasma. 


\section{Seeing QCD first-order phase transition with phi mesons}

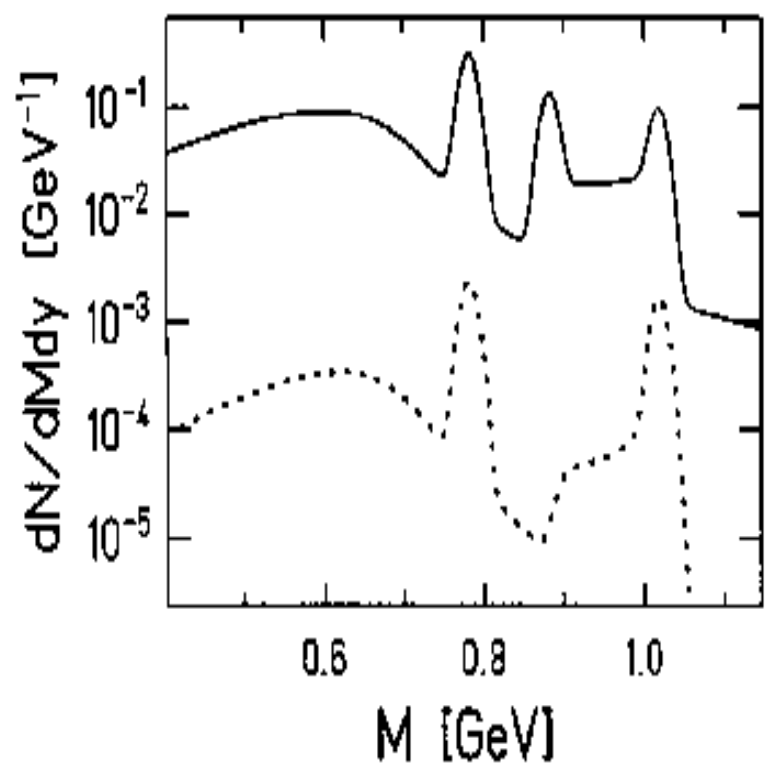

Figure 7: The dilepton invariant mass spectrum in heavy ion collisions. The solid line is obtained with a first-order phase transition while the dotted line is from the scenario without the quark-gluon plasma. From Ref.[69].

Another possible way to verify the existence of a first-order QGP to hadronic matter phase transition in heavy ion collisions is to study phi meson production through its emitted dileptons. Because of its narrow width of $\sim 4 \mathrm{MeV}$, a phi meson in heavy ion collisions normally decays to dileptons mainly at the time when it freezes out from the hadronic matter. If there is a first-order QGP to hadronic matter phase transition, dileptons from phi mesons decay during the mixed phase of the first-order phase transition can also be appreciable as a result of the long duration of the mixed phase. Since the phi meson mass may be reduced in hot and dense medium, the emitted dileptons from phi mesons in the mixed phase thus have lower invariant masses than those from phi mesons decay at freeze out. As a result, the dilepton invariant mass spectra would have an additional peak at a lower mass than that corresponding to the free phi mass mass. In Ref. [69], this effect is studied in a hydrodynamic model for heavy ion collisions that includes a first-order phase transition. With a temperature-dependent phi meson mass determined from the QCD sum rule [70], the resulting phi meson invariance spectrum indeed shows another peak between the $\rho / \omega$ and free phi meson peaks as shown by the solid line in Fig. 7. Although the double phi peak feature in the dilepton invariant mass spectrum would remain if the quark-gluon plasma to hadronic matter phase transition is a crossover but lasts a sufficient long time [71], it becomes a shoulder in the absence of the quark-gluon plasma as shown by the dashed line in the figure. Since the flow velocity is smaller at phase transition than at hadronic freeze out, the inverse slope parameter of the low mass phi meson transverse momentum spectrum is also expected to be smaller than that of 
the transverse momentum spectrum of normal phi mesons and thus reflects more closely the phase transition temperature.

\section{Conclusions}

Heavy ion collisions in the low-energy runs at RHIC and at FAIR is expected to produce a quark-gluon plasma that has an appreciable baryon chemical potential, allowing thus the possibility to study the location of the critical endpoint in the QCD phase diagram, where the quark-gluon plasma to hadronic matter transition changes from a crossover to a first-order phase transition. Using a multiphase transport model, that includes interactions in both initial partonic and final hadronic matters and the transition between these two phases of matter via a quark coalescence model, we have studied the effect of partonic interactions on the elliptic flows of identified hadrons as well as the effect of density fluctuations due to a first-order transition between the quark-gluon plasma and hadronic matter on fluctuations of hadron mean transverse momentum and produced deuteron number and on two-pion correlations. We have found that strong partonic interactions during the partonic stage of the collisions enhance significantly the particle elliptic flow and also leads to an approximate constituent quark number scaling of the elliptic flows of identified hadrons. Although the presence of density fluctuation due to a first-order phase transition has little effect on the fluctuation of final hadron mean transverse momentum, it affects appreciably the yield of produced deuterons and the width of the two-pion correlation function. We have further found that the net baryon-rich quark-gluon plasma formed in these collisions provides the possibility to test the resonance scattering mechanism for charm energy loss in quark-gluon plasma, which has been quite successful in understanding the charm energy loss and elliptic flow in heavy ion collisions at RHIC, i.e., a larger energy loss of anti-charm quarks than quarks in heavy ion collisions at lower energies. The long duration expected for a first-order phase transition can also lead to the appearance of a lower mass peak between the $\rho / \omega$ and $\phi$ mesons in the dilepton invariance mass spectrum as a result of the lower phi meson mass in the mixed phase of the quark-gluon plasma to hadronic matter transition. Many interesting phenomena are thus expected to be observed in RHIC low-energy runs and at FAIR, and they not only are of their own intrinsic interest but will also provide the possibility to study the location of the critical endpoint in the QCD phase diagram, where the crossover transition at low baryon chemical potential changes to a first-order transition when the baryon chemical becomes sufficient large.

\section{Acknowledgements}

This talk was based on work supported by the US National Science Foundation under Grant Nos. PHY-0758115 and the Welch Foundation under Grant No. A-1358 (C.M.K.), the NNSF of China under Grant Nos. 10405011 (B.W.Z), 10575071 and 10675082, MOE of China under project NCET-05-0392, Shanghai Rising-Star Program under Grant No. 06QA14024, and the SRF for ROCS, SEM of China (L.W.C.).

\section{References}

[1] E. Shuryak, Phys. Lett. B 78, 150 (1978). 
[2] J. Rafelski and B. Müller, Phys. Lett. B 101, 111 (1982).

[3] S. Pratt, Phys. Rev. Lett. 53, 1219 (1984); Phys. Rev. D 33, 1314 (1986).

[4] G. F. Bertsch, Nucl. Phys. A 498, 173c (1989).

[5] T. Matsui and H. Satz, Phys. Lett. B 178, 416 (1986).

[6] J.Y. Ollitrault, Phys. Rev. D 46, 229 (1992).

[7] X. N. Wang and M. Gyulassy, Phys. Rev. Lett. 68, 1480 (1992).

[8] S. Voloshin, Nucl. Phys. A 715, 379 (2003).

[9] P. Braun-Munzinger, D. Majestra, K. Redlich, and J. Stachel, Phys. Lett. B 518, 41 (2001).

[10] G. Torrieri and J. Rafelski, J. Phys. G 30, 557 (2004).

[11] D. Teaney, J. Lauret and E. V. Shuryak, Phys. Rev. Lett. 86, 4783 (2001); Nucl. Phys. A 698, 479 (2002).

[12] P. Huovinen, P. F. Kolb, and U. Heinz, Nucl. Phys. A 698, 475 (2002).

[13] T. Hirano and K. Tsuda, Phys. Rev C 66, 054905 (2002).

[14] B. Zhang, C. M. Ko, B. A. Li and Z. W. Lin, Phys. Rev. C 61, 067901 (2000).

[15] Z. W. Lin, S. Pal, C. M. Ko, B. A. Li, and B. Zhang, Phys. Rev. C 64, 011902 (2001); Nucl. Phys. A 698, 375 (2002).

[16] Z. W. Lin, C. M. Ko, B. A. Li, B. Zhang and S. Pal, Phys. Rev. C 72, 064901 (2005).

[17] D. Molnar and M. Gyulassy, Nucl. Phys. A 697, 495 (2002) [Erratum-ibid. A 703, 893 (2002)].

[18] W. Cassing and E. L. Bratkovskaya, Phys. Rep. 308, 65 (1999).

[19] S. Bass et al., Prog. Part. Nucl. Phys. 41, 225 (1998).

[20] B. H. Sa, X. Cai, Z. D. Su, A. Tai, and D. M. Zhou, Phys. Rev. C 66, 044902 (2002).

[21] D. M. Zhou, X. M. Li, B. G. Dong, B. H. Sa, Phys. Lett. B 638, 461 (2006).

[22] L. Bravina, K. Tywoniuk, E. Zabrodin, G. Burau, J. Bleibel, C. Fuchs, and A. Faessler, Phys. Lett. B 631, 109 (2005).

[23] Z. Xu and C. Greiner, Phys. Rev. C 71, 064901 (2005).

[24] R. Hwa and C. B. Yang, Phys. Rev. C 67, 034902 (2003).

[25] V. Greco, C. M. Ko, and P. Lévai, Phys. Rev. Lett. 90, 202302 (2003); Phys. Rev. C 68, 034904 (2003).

[26] R. J. Fries, B. Müller, C. Nonaka, and S. A. Bass, Phys. Rev. Lett. 90, 202303 (2003); Phys. Rev. C 68, 044902 (2003).

[27] Z. W. Lin and D. Molnar, Phys. Rev. C 68, 044901 (2003).

[28] M. Gyulassy and X. N. Wang, Nucl. Phys. B 420, 583 (1994).

[29] M. Gyulassy, P. Levai, and I. Vitev, Phys. Rev. Lett. 85, 5535 (2001).

[30] J. Adams et al. [STAR Collaboration], Nucl. Phys. A 757, 102 (2005).

[31] M. A. Stephanov, PoS Lat2006:024 (2006). 
[32] B. Zhang, C. M. Ko, B. A. Li, Z. W. Lin, and B. H. Sa, Phys. Rev. C 62, 054905 (2000).

[33] B. Zhang, C. M. Ko, B. A. Li, Z. W. Lin, and S. Pal, Phys. Rev. C 65, 054909 (2002).

[34] C. M. Ko, Z. W. Lin, and S. Pal, Heavy Ion Phys. 17, 219 (2003).

[35] S. Pal. C. M. Ko, and Z. W. Lin, Nucl. Phys. A 730, 143 (2004).

[36] X. N. Wang and M. Gyulassy, Phys. Rev. D 44, 3501 (1991).

[37] B. Zhang, Comput. Phys. Commun. 109, 193 (1998).

[38] T. Sjostrand, Comput. Phys. Commun. 82, 74 (1994);

[39] B. A. Li and C. M. Ko, Phys. Rev. C 52, 2037 (1995).

[40] B.A. Li, A.T. Sustich, B. Zhang, and C.M. Ko, Int. Jour. Phys. E 10, 267-352 (2001).

[41] D. Kharzeev and M. Nardi, Phys. Lett. B507, 121 (2001).

[42] Z. W. Lin and C. M. Ko, Phys. Rev. C 65, 034904 (2002).

[43] L. W. Chen, C. M. Ko and Z. W. Lin, Phys. Rev. C 69, 031901 (2004)

[44] Z. W. Lin, C. M. Ko and S. Pal, Phys. Rev. Lett. 89, 152301 (2002).

[45] K. H. Ackermann et al. [STAR Collaboration], Phys. Rev. Lett. 86, 402 (2001).

[46] J. Adams et al. [STAR Collaboration], Phys. Rev. lett. 92, 062301 (2004).

[47] C. Adler et al. [STAR Collaboration], Phys. Rev. Lett. 87, 082301 (2001).

[48] Z. W. Lin and C. M. Ko, J. Phys. G 30, S263 (2004).

[49] L. W. Chen, V. Greco, C. M. Ko and P. F. Kolb, Phys. Lett. B 605, 95 (2005).

[50] L. W. Chen and C. M. Ko, Phys. Lett. B 634, 205 (2006).

[51] L. W. Chen and C. M. Ko, Phys. Rev. C 73, 014906 (2006).

[52] B. Zhang, L. W. Chen, and C. M. Ko, Phys. Rev. C 72, 024906 (2005).

[53] L. W. Chen and C. M. Ko, Phys. Rev. C, 73, 044903 (2006).

[54] S. S. Adler et al. [PHENIX Collaboration], Phys. Rev. Lett. 96032301 (2006).

[55] J. Bielcik and STAR Collaboration, Nucl. Phys. A 774, 697 (2006).

[56] H. van Hees, V. Greco, and R. Rapp, Phys. Rev. C 73, 034913 (2006).

[57] V. Greco and C. M. Ko, Phys. Lett. B 595, 202 (2004).

[58] A. Onishi and J. Randrup, Phys. Lett. B 394, 260 (1997).

[59] L. W. Chen, C. M. Ko, and B. A. Li, Phys. Rev. C 68, 017601 (2003); Nucl. Phys. A 729, 809 (2003).

[60] G. Bertsch, M. Gong and M. Tohyama, Phys. Rev. C 37, 1896 (1988).

[61] S. Pratt, T. Csörgö and J. Zimanyi, Phys. Rev. C 42, 2646 (1990).

[62] C. Adler et al. [STAR Collaboration], Phys. Rev. Lett. 87, 082301 (2001).

[63] S. C. Johnson [PHENIX Collaboration], Nucl. Phys. A 698, 603 (2002).

[64] K. Adcox et al. [PHENIX Collaboration], Phys. Rev. Lett. 88192302 (2002).

[65] S. Afanasiev et al. [PHENIX Collaboration], Phys. Rev. Lett. 100, 232301 (2008). 
[66] B. Tomasik and U. W. Heinz, arXiv:nucl-th/9805016.

[67] S. Pratt et al., Nucl. Phys. A 566, 103c (1994).

[68] T. Sjorstrand, Comput. Phys. Commun. 82, 74 (1994).

[69] M. Asakawa and C. M. Ko, Phys. Lett. B 322, 33 (1994).

[70] M . Asakawa and C. M. Ko, Nucl. Phys. A 572, 732 (1994).

[71] M. Asakawa and C. M. Ko, Phys. Rev. C 50, 3064 (1994). 